\title{
Rehabilitation of Completely Edentulous Patient With Implant Supported Full Arch Fixed Prosthesis
}

Pratyusha Lakshmi K ${ }^{1}$, Ravi Kumar $C^{2}$, Sunil G3 , Chalapathi Rao D4

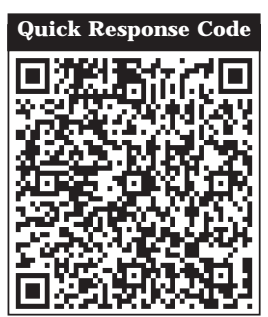

doi: $10.5866 / 2015.7 .10073$

${ }^{1}$ Post Graduate

2Prof \& Head

3\&4Reader

Department of Prosthodontics and Crown and Bridge, Mamata Dental College and Hospital, Khammam, Telangana State, India.

\section{Article Info:}

Received: J anuary 8, 2015

Review Completed: February 9, 2015

Accepted: March 10, 2015

Available Online: April, 2015 (www.nacd.in)

(c) NAD, 2015 - All rights reserved

Email for correspondence:

pratyushakoganti@gmail.com

\begin{abstract}
:
Loss of natural teeth results in both aesthetic and functional deficits as the age of the patient advances. This leads to significant reduction in the patient's qual ity of life and self image perception. Therefore offering the correct treatment options to the patients losing their teeth either due to extraction or as a natural physiologic process is an important aspect of comprehensive patient treatment. Fixed or removable implantsupported restorations are among the prosthesis designs used for the treatment of the edentulous mouth. Planning of the treatment steps and designing of the implant-supported fixed final prosthesis are primarily important to respond theaesthetic and functional requirements of the fully edentul ous patient. This case report tries to explain in detail the step-by-step procedure in treatment planning, surgical and prosthetic aspects taken to completely rehabilitate a 70-year-old completely edentulous patient using an implant supported fixed prosthesis. A total of 12 endosteal dental implants were placed in both jaws followed by fabrication of implant supported full arch fixed dentures.
\end{abstract}

Key words: I mplant-supported prosthesis, surgical template, Metal-ceramic, Fixed prostheses, Treatment planning.

\section{INTRODUCTION:}

The treatment for edentulism is well documented by surgical placement of dental implants. Treatment success rates are high and postoperative complications relatively modest. ${ }^{1}$ Implant supported prosthodontic rehabilitation of total edentulism remains one of the most complex restorative challenges because of the number of variables that affect both the aesthetic and functional aspects of the prosthesis. Among the prosthesis designs used for the treatment of the edentulous mouth are fixed or removable implant- supported restorations. Since the aesthetic requirements and preoperative situation of each patient varies, considerable time should be devoted on accurate diagnosis to ensure patient desires are satisfied and predictable outcomes are achieved. ${ }^{2}$ When deciding whether to use a fixed or removable implant-supported full-arch restoration, a multitude of factors should be considered. . $^{3,4}$

After achieving the reliable osseointegration; sequential procedure of recording the final impressions, maxillo-mandibular relation records, fabrication and try-in of the framework, 
confirmation of the maxillo-mandibular relation by using a second record and ceramic veneering of the framework; were mentioned as a classical protocol for such restorations. ${ }^{5,6}$ Obtaining a proper occlusion and providing a functioning articulation in a suitable relationship with the dento-facial esthetics are mentioned as the other challenges in constructing full-mouth implant-supported fixed prostheses. ${ }^{7}$

In view of all the above considerations, the aim of this paper is to report the design of a full-mouth implant supported metal ceramic fixed prostheses of a completely edentulous patient.

\section{CASE REPORT}

A 70-year-old male patient reported to Department of Prosthodontics, Mamata Dental College, Khammam, with the chief complaint of missing teeth in the upper and lower front and back tooth region, and difficulty in mastication. After initial consultation, extraoral examination revealed collapsed facial profile, deepened naso-labial and mento-labial fold. Intraoral examination revealed the presence of completely edentulous upper and lower arches, with moderately resorbed maxillary arch in the first and second molar regions. During the initial visit, appointment was scheduled for complete medical examination, lateral cephalometric radiograph, panoramic radiograph, and impressions for study models, maxillomandibular relations and face bow transfer. Medical and haematological examinations were found to be normal.

Panoramic radiograph revealed closed proximity of the sinus floor to the alveolar ridge (Figurel and 2). Complete intraoral condition was analyzed and different treatment options ranging from conventional removable prosthesis to two-stage implant placement with sinus lift in the maxillary posterior region was planned and discussed with the patient. The patient was unwilling for invasive surgical procedures. In view of the above said considerations a fixed prosthesis including implant placement till the second premolar region in the maxillary and mandibular arches was planned. Pair of templates were fabricated on the master cast and the patient was subjected to CBCT with the template in situ in order to select the desired length and diameter of theimplants. The preoperative planning was conducted with a diagnostic wax up to have an idea of the treatment objective. This model was duplicated to fabricate surgical stent that would serve as reference during surgical procedures for implant placement.

\section{PROCEDURE :}

\section{Phase 1:}

The assessment of Cone Beam Computer Tomography and orthopantomograph did not reveal any bony defects in the sites chosen for implant placement. Vital signs werechecked and consent had been obtained from the patient prior to the surgery. Patient was advised for prophylactic antibioticsi.e., Amoxicillin $1 \mathrm{~g} / 1$ hour prior to the surgical procedure. Extraorally the patient was disinfected with $5 \%$ povidone iodine paint and rinsed with $2 \%$ povidone iodine for $30 \mathrm{sec}$. Local anesthesia was administered using lignocaine $2 \%$ adrenaline. Crestal incision was made $2 \mathrm{~mm}$ away from the midcrestal region towards the palatal and lingual side using no. 15 bard parker blade and full-thickness mucoperiosteal flaps were elevated. Initial osteotomy preparation was started with a round bur followed by a pilot drill and consecutively enlarged up to $3.3 \mathrm{~mm}$ to $4.2-\mathrm{mm}$ diameter. Root form endosseous implants weretorque in to their planned position and cover screws were placed. A check OPG was taken to verify the parallelism and depth (Figure 3), the flaps were approximated with 3-0 vicryl sutures (vicryl*plus anti bacterial sutures, ETHICON, Inc, India). Patient was kept on antibiotics and analgesics for 7 days and recalled every 2 days for irrigation of the suture site.

\section{Phase 2:}

After a healing period of 3 months (Figure 4), crestal incisions were made in the maxillary and mandibular arches and a mucoperiosteal flap was el evated to expose the cover screws. After retrieval of cover screws, gingival formers were placed over the respective implants and the mucoperiosteal flap was sutured in order to achieve a healthy gingival collar. The healing abutments were unscrewed after a period of 1 week exhibitig a freshly formed healthy gingival collar (Figure 5).

\section{Phase 3:}

Maxillary and mandibular final impressions were made with poly vinylsiloxane elastomeric impression material by using closed tray impression technique. I mplant anal ogs fixed to the abutments, were placed into their own replicas and the impressions were poured with type IV dental stone 


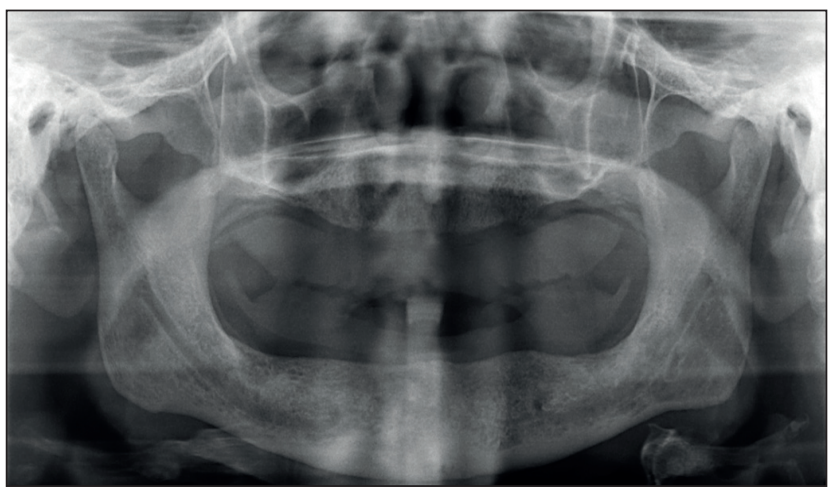

Figurel: Pre-operative OPG showing close proximity of the sinus floor in the maxillary posterior quadrants.

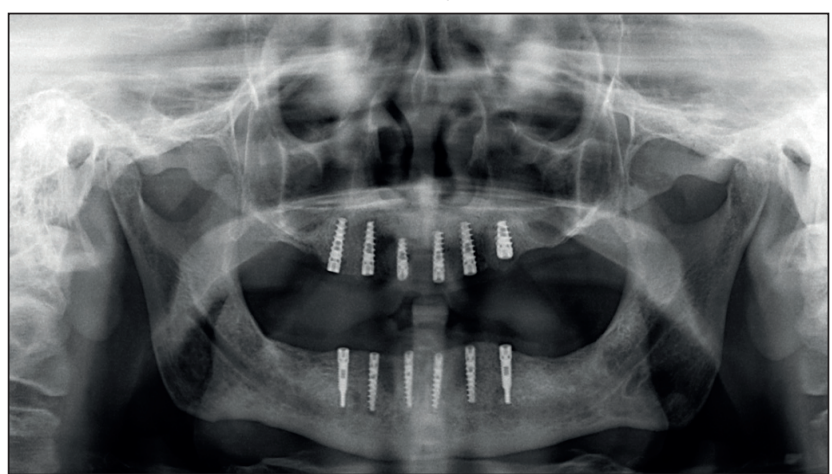

Figure 3: I mmediate postoperative OPG

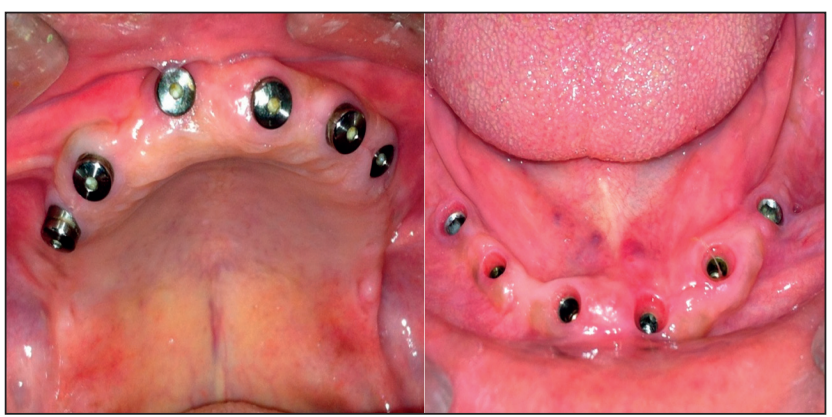

Figure 5: One week after placement of healing abutments. Maxillary arch (left image) and mandibular arch (right image).

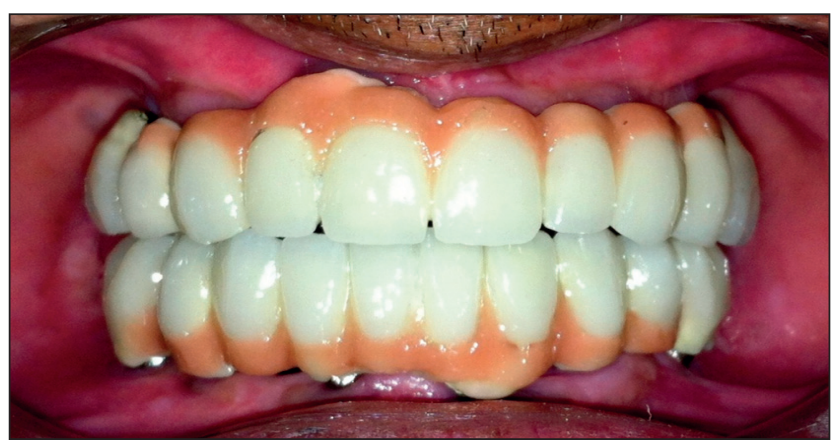

Figure 7: Fabrication of a $\mathrm{n}$ implant supported fixed full arch prosthesis in the maxillary arch (left image) and mandibular arch (right image) - facial view

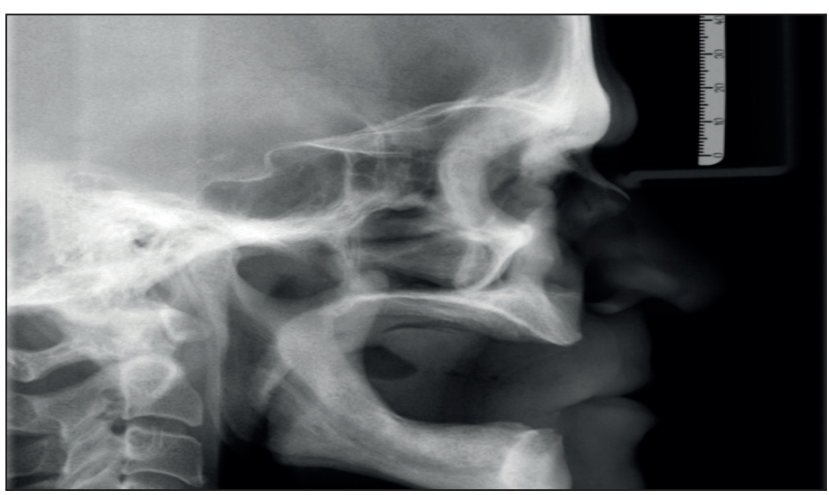

Figure 2: Pre-operative lateral cephal ometric radiograph.

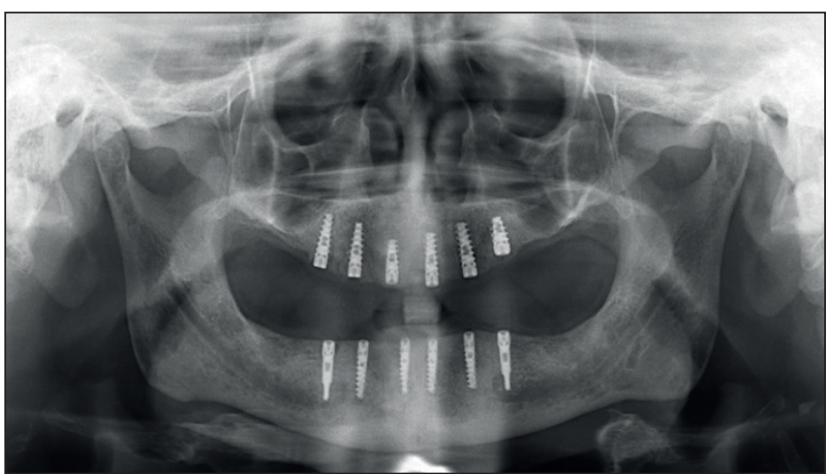

Figure 4: Post operative OPG after three months of healing period.

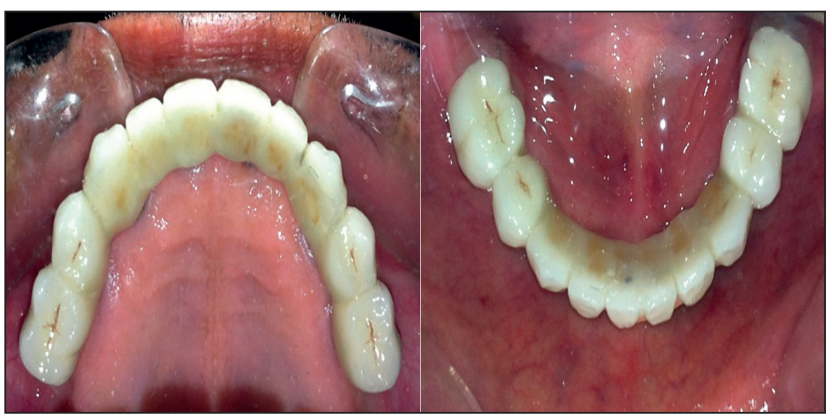

Figure 6: Fabrication of an implant supported fixed full arch prosthesis in the maxillary arch (left image) and mandibular arch (right image) - occlusal view.

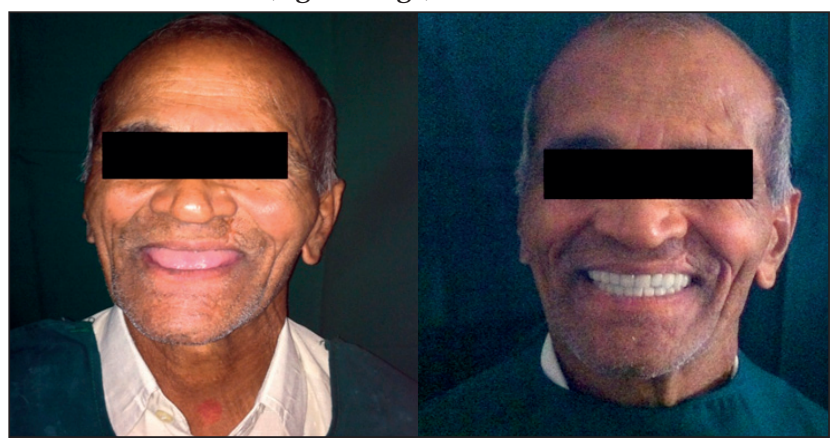

Figure 8: Pre-operative (left side image) and post-operative (right side image) of the patient. 
in order to obtain master casts. Denture bases were fabricated from autopolymerizing methacrylate material. Buccal flanges were used to check the fitting of the denture base but labial flanges were sectioned in order to avoid the excessive lip support. In order to provide relief, perforations were made at corresponding implant sites on the respective denture bases. Occlusal rims were fabricated on the denture bases with modelling wax, and the maxillomandibular relations were obtained and transferred to a semi-adjustable articulator. Teeth arrangement was done in order to demonstrate the dimensions, location, position and occlusion of the forthcoming fixed prostheses and patients consent was taken. The centric relation, intercuspal position and vertical dimension were also transferred to the articulator from the patient, with the help of polyvinyl siloxane putty index. During metal trial, fit of the castings and occlusal clearance were checked. A bisquetrial was doneto confirm fit, shade and occlusal parameters and later glazed. The restorations were cemented with an intermediate restorative material to verify its performance for 15 days and later luted with Type I glass ionomer cement (Figure 6,7 and 8).

\section{DISCUSSION}

Full-arch implant-supported fixed dental prostheses are well-documented therapeutic applications for completely edentulous patients. ${ }^{8}$ Planning of the treatment steps and designing of the final prosthesis are primarily important steps in rehabilitation of such prosthesis. In maxillary molar region implant placement was ruled out due to the close proximity of the sinus floor and the patient's unwillingness for invasive surgical procedures. So, a cantilever extension of a single molar was fabricated in both maxillary and mandibular arches excluding the second premolar. The success of these restorations is linked to patientrelated factors such as maintenance of proper oral hygiene and eating habits, and treatment planrelated factors such as occlusal adjustment and stability. The implant-supported fixed dental prostheses (FDPs) are a safe and predictable treatment method with high survival rates. However, biological and technical complications were frequent (33.6\%). To minimize the incidence of complications, dental professionals should make great effort in choosing reliable components and materials for implant-supported FDPs and the patients should be advocated well-structured maintenance system after treatment. ${ }^{9}$

\section{CONCLUSION:}

Extensive preoperative planning and treatment coordination are necessary for treatment success. I maging tools, a diagnostic wax-up and a surgical guide, along with a good understanding of anatomy and surgical principles are essential. Early recognition of problem etiology and prompt treatment may prove to be invaluable to clinicians. Within the limitation of this case, clinical results seem to support the case that CT-based software and laboratory based surgical guides may be used to decrease the incidence of implant-associated complications and to better assist the clinician in selecting and applying the most appropriate treatment options.

\section{REFERENCES:}

1. Branemark PI, Svensson B, van Steenberghe D. Ten-year survival rates of fixed prostheses on four or six implants and modum Branermark in full edentulism. Clin Oral I mplants Res 1995; 6:227-231.

2. J ivraj $\mathrm{S}, \mathrm{Chee} \mathrm{W}$, Corrado $\mathrm{P}$. Treatment planning of the edentulous maxilla. Br Dent J 2006; 201:261-279.

3. Zafiropoulos GG, H offman O. I mplant-retained dentures for full-arch rehabilitation: a case report comparing fixed and removable restorations. Gen Dent 2011; 59:137-143.

4. Barao VA, Delben J A, Lima J, Cabral T, Assunçao WG. Comparison of different designs of implant-retained overdentures and fixed full-arch implant-supported prosthesis on stress distribution in edentulous mandiblea computed tomography-based three-dimensional finite element analysis. J Biomech 2013; 46:1312-1320.

5. Proussaefs P. Clinical and laboratory steps for the fabrication of a fixed, cement-retained, implant-supported, complete-arch maxillary prosthesis. Int J Periodontics Restorative Dent 2004; 24:344-351.

6. Rosenbaum N. Full-arch implant-retained prosthetics in general dental practice. Dent Update 2012; 39:108-110.

7. Klineberg IJ, Trulsson M, Murray GM. Occlusion on implants - is there a problem? J Oral Rehabil 2012; 39:522537.

8. Calvani L, Michalakis K, Hirayama H. The influence of fullarch implant-retained fixed dental prostheses on upper lip support and lower facial esthetics: preliminary clinical observations. Eur J Esthet Dent 2007; 2:420-428.

9. Pjetursson BE, Thoma D, J ung R, Zwahlen M, Zembic A. A systematic review of the survival and complication rates of implant-supported fixed dental prostheses (FDPs) after a mean observation period of at least 5 years. Clin Oral Implants Res 2012; 6:22-38. 